\title{
Erratum to: OrthoFiller: utilising data from multiple species to improve the completeness of genome annotations
}

Michael P. Dunne and Steven Kelly

\section{Erratum}

After publication of the original article [1] the authors found the following errors had occurred:

1. Saccaromyces should be spelt Saccharomyces in Table 1. (Table 1)

2. Kluveromyces should be spelt Kluyveromyces in Table 1. (Table 1)

3. Table 4, columns 3, 5, 7 and 9: All values within these columns should be placed in brackets to indicate the standard deviation. (Table 4)

4. The legend for Table 4 should read: 'Numbers shown are rounded mean values from 5 disjoint removed subsets of genes, with standard deviations bracketed and not ' 10 removed subsets' as per the original article. (Table 4)

The original article has been corrected.

Corrected versions of all figures and tables are included in this Erratum:

Corrected Table 1

Table 1 Species Set A, fungal species used for algorithm validation

\begin{tabular}{|c|c|c|c|c|}
\hline Species Name & Source & Strain & Taxonomy ID & References \\
\hline Eremothecium gossypii & $\left.J G\right|^{a}$ & ATCC10895 & 284,811 & [12] \\
\hline Debaromyces hansenii & $J G l$ & CBS767 & 284,592 & [13] [14] \\
\hline Kluyveromyces lactis & $J G l$ & CLIB210 & 284,590 & [13] \\
\hline Saccharomyces cerevisiae & $S G D^{b}$ & S288C & 559,292 & {$[24]$} \\
\hline Yarrowia lipolytica & $J G l$ & CLIB122 & 284,591 & [13] \\
\hline
\end{tabular}

ajoint Genome Institute; 'baccharomyces Genome Database

* Correspondence: steven.kelly@plants.ox.ac.uk

Department of Plant Sciences, University of Oxford, South Parks Road, Oxford OX1 3RB, UK 


\section{Corrected Table 4}

Table 4 Recovery of removed genes in A. thaliana, averaged over five runs

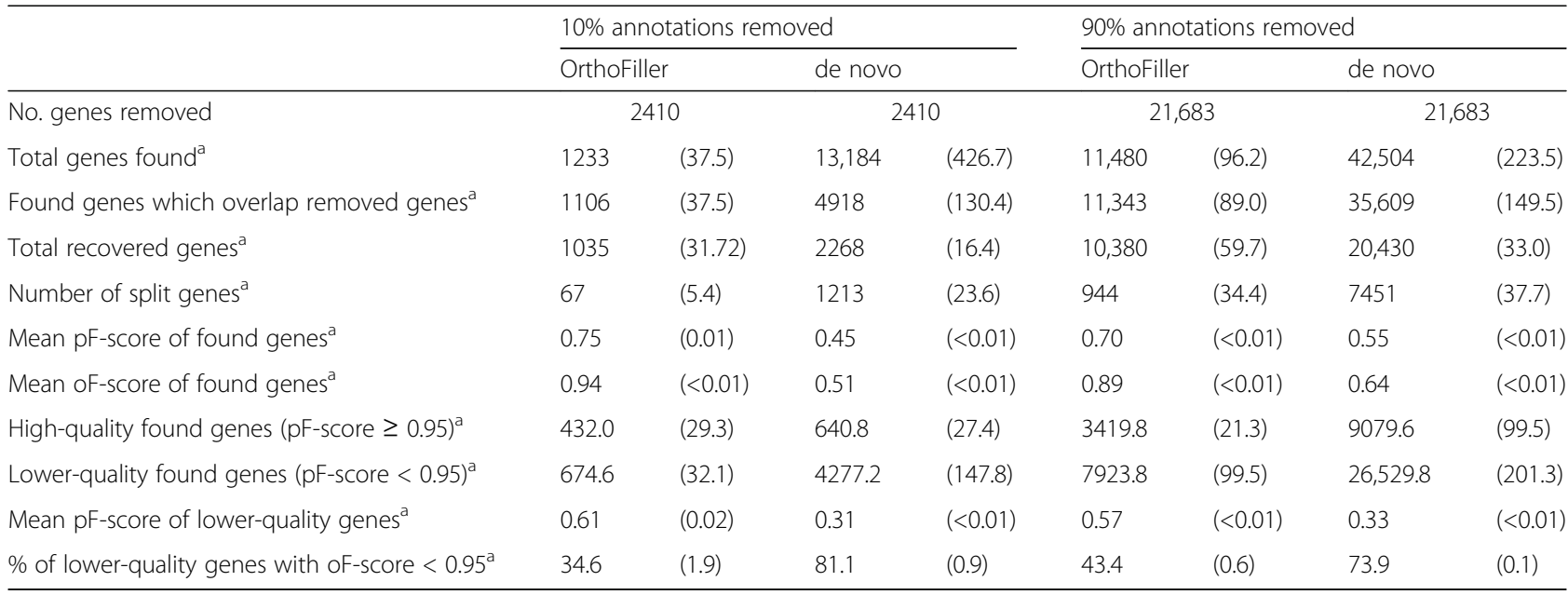

${ }^{a}$ Numbers shown are rounded mean values from 5 disjoint removed subsets of genes, with standard deviations bracketed

Received: 7 June 2017 Accepted: 7 June 2017

Published online: 27 June 2017

\section{Reference}

1. Dunne M, Kelly S. OrthoFiller: utilising data from multiple species to

improve the completeness of genome annotations. BMC Genomics. 2017;

18:390. doi:10.1186/s12864-017-3771-X. 